\title{
Manipulation of interfacial instabilities by using a soft, deformable solid layer
}

\author{
GAURAV $^{1}$ and V SHANKAR ${ }^{2, *}$ \\ ${ }^{1}$ Indian Institute of Technology Roorkee, Roorkee, India \\ ${ }^{2}$ Indian Institute of Technology Kanpur, Kanpur, India \\ e-mail: goravfch@iitr.ac.in; vshankar@iitk.ac.in
}

MS received 31 August 2014; revised 25 December 2014; accepted 9 January 2015

\begin{abstract}
Multilayer flows are oftensusceptible to interfacial instabilities caused due to jump in viscosity/elasticity across thefluid-fluid interface. It is frequently required to manipulate and control these interfacial instabilities in various applications such as coating processes or polymer coextrusion. We demonstrate here the possibility of using a deformable solid coating to control such interfacial instabilities for various flow configurations and for different fluid rheological behaviors. In particular, we show complete suppression of interfacial flow instabilities by making the walls sufficiently deformable when the configuration was otherwise unstable for the case of flow past a rigid surface. While these interfacial instabilities could be suppressed in certain parameter regimes, it is also possible to enhance the flow instabilities by tuning the shear modulus of the deformable solid coating for other ranges of parameters.
\end{abstract}

Keywords. Interfacial instabilities; manipulation and control; deformable solid.

\section{Introduction}

The flow of multiple immiscible liquid layers involving one or more fluid-fluid interfaces is encountered in various technologically important processes, for example, in production of laminated sheets, coating processes, lubricated pipelining, coextrusion of polymers, and more recently in microfluidic devices to produce uniform sized microdrops and bubbles. These multilayer flows are prone to several interfacial instabilities arising due to jump in either viscosity or elasticity across the fluid-fluid interface (Yih 1967; Chen 1991). These interfacial instabilities are undesirable in applications like coating or polymer coextrusion where a smooth interface between different liquid layers is required to impart unique mechanical and optical properties. On the other hand, such interfacial instabilities are the primary mechanism that breaks a liquid column into smaller droplets (Rayleigh 1878, 1892) and hence, are responsible for the creation of microdrops and emulsions in microfluidic devices. Thus, a key aspect in multilayer flows is the ability to control and manipulate interfacial instabilities to meet the finished product

${ }^{*}$ For correspondence 
requirements. In the present work, we demonstrate the control and manipulation of interfacial instabilities by using a soft, deformable solid coating for a variety of geometric configurations and for different fluid rheological behavior.

When a fluid flows past a flexible surface, the dynamics of fluid and solid are coupled via velocity and stress continuity conditions at fluid-solid interface. It has been now well established, by a series of theoretical and experimental studies, that such elastohydrodynamic coupling induces new fluid-solid interfacial instabilities both at zero and finite Reynolds number which are qualitatively very different from those present in flow past rigid surfaces (Kumaran 2000; Verma \& Kumaran 2013). All these studies illustrated that the presence of deformable fluid-solid interface renders flow unstable at Reynolds number which is much lower than that observed in case of flow past rigid surfaces. In contrast to above mentioned studies, Shankar and coworkers investigated the effect of including a deformable solid coating on the instability of fluid-fluid interface arising due to viscosity/elasticity contrast in case of flow of multiple liquid layers for a diverse class of geometrical and physical settings (Shankar 2005; Jain \& Shankar 2007, 2008; Gaurav \& Shankar 2010a, 2013). These studies demonstrated that it is possible to completely suppress the interfacial instabilities by tuning the deformability of the solid layer when the instability existed for the case of flow past rigid surface. It was also shown that it is possible to even destabilize the fluid-fluid interface when the interface otherwise remains stable in rigid limit. In the present paper, we summarize these findings related to the manipulation and control of interfacial instabilities by using a deformable solid coating.

A common feature of all the configurations considered in the present work is the presence of a liquid-liquid (LL) and/or a gas-liquid (GL) interface which undergo instabilities due to jump in properties across the interface. The stability characteristics depend strongly on the geometric configuration as well as on the rheological properties of the fluids. For example, inertia is required to destabilize the GL free surface in case of a single Newtonian liquid film falling down an incline (Yih 1963), or to destabilize the LL interface for the case of flow of two immiscible Newtonian liquid layers in a rectangular channel (Yih 1967). On the other hand, multiple Newtonian liquid films falling down an incline (Chen 1993) or plane Couette flow of two viscoelastic liquid layers become unstable even in creeping flow limit (Renardy 1988). The presence of cylindrical fluid-fluid interface introduces an additional surface tension induced capillary instability along with instability present due to viscosity/elasticity contrast (Preziosi et al 1989). Various strategies like imposing in-plane oscillations for the case of single and two layer liquid film flows (Lin \& Chen 1997; Huang \& Khomami 2001), imposing a temperature gradient along the wall (Demekhin et al 2006), and adding insoluble surfactants at LL or GL interface (see for example, Bassom et al (2012)) have been investigated to explore the possibility of controlling interfacial instabilities in different settings. While these studies have suggested 'active' methods (i.e. externally imposed oscillations or heating of the plate) toward suppressing the fluid-fluid instabilities, here, we examine the feasibility of a 'passive' method, where a soft solid coating is proposed for suppressing the interfacial instabilities in different flow situations.

\section{Methodology}

The flow configurations considered here consist of one or more fluid layers and a deformable solid layer. The fluid layers are modeled either as Newtonian fluid or viscoelastic fluid represented by an Oldroyd-B model. The total stress tensor for the fluid layer is $\mathbf{T}=-p \mathbf{I}+\boldsymbol{\tau}$, where $\boldsymbol{\tau}=\mu\left[\nabla \mathbf{v}+(\nabla \mathbf{v})^{T}\right]$ for a Newtonian liquid with viscosity $\mu$. For a viscoelastic Oldroyd-B fluid model, $\boldsymbol{\tau}$ is referred as extra-stress tensor comprising of a polymeric contribution $\left(\tau^{\boldsymbol{p}}\right)$ and 
a Newtonian solvent contribution $\left(\tau^{s}\right): \tau=\tau^{p}+\tau^{s}$. The polymeric contribution is given by upper convected Maxwell equation:

$$
\tau_{R}\left[\frac{\partial \boldsymbol{\tau}^{p}}{\partial t}+\mathbf{v} \cdot \nabla \boldsymbol{\tau}^{p}-(\nabla \mathbf{v})^{T} \cdot \boldsymbol{\tau}^{p}-\boldsymbol{\tau}^{p} \cdot \nabla \mathbf{v}\right]+\boldsymbol{\tau}^{p}=\mu_{p}\left[\nabla \mathbf{v}+(\nabla \mathbf{v})^{T}\right],
$$

while the solvent is considered to be a Newtonian solvent: $\boldsymbol{\tau}^{s}=\mu_{\text {sol }}\left[\nabla \mathbf{v}+(\nabla \mathbf{v})^{T}\right]$ In the above equations for Oldroyd-B model, $\mu_{p}$ is the polymer contribution to the shear viscosity, $\tau_{R}$ is the relaxation time characterizing the elasticity of the fluid and $\mu_{s o l}$ is the solvent viscosity. The governing equations for fluid and solid layers are nondimensionalized by using the following scales: the total thickness of fluid layer(s) $R$ for lengths, $V$ as characteristic velocity and $\mu V / R$ for stresses and pressure. The dimensionless governing equations for liquid layer are

$$
\nabla \cdot \mathbf{v}=0, \quad \operatorname{Re}\left[\frac{\partial \mathbf{v}}{\partial t}+\mathbf{v} \cdot \nabla \mathbf{v}\right]=\nabla \cdot \mathbf{T}+\frac{\rho \mathbf{g} R^{2}}{\mu V}
$$

$\mathbf{v}$ represents the velocity field in the liquid, $R e=\rho V R / \mu$ is the Reynolds number, and the dimensionless Cauchy stress tensor for Newtonian liquid is $\boldsymbol{\tau}=\left[\nabla \mathbf{v}+(\nabla \mathbf{v})^{T}\right]$. For Oldroyd-B fluid model, two nondimensional parameters appear, namely, Weissenberg number $W=\tau_{R} V / R$ which is a measure of elasticity or relaxation time of fluid, and a ratio of solvent to total viscosity $S=\mu_{\text {sol }} /\left(\mu_{\text {sol }}+\mu_{p}\right)$.

The deformable solid layer is modeled using a simple nonlinear neo-Hookean solid model which captures the behavior of real soft, deformable materials like rubber reasonably well (Macosko 1994). We use a Lagrangian framework to write the governing equations for solid and Eulerian description for writing down the governing equations for fluid layer(s). In Lagrangian description, spatial positions in reference (unstressed) configuration denoted by $\mathbf{X}=(X, Y, Z)$ are used as independent variables to describe the dynamics of neo-Hookean deformable solid layer. The current spatial position is denoted by $\mathbf{x}=x, y, z$ and forms the independent variable in Eulerian description for fluid layers. The dimensionless Cauchy stress tensor for neo-Hookean solid is $\sigma=-\hat{p_{s}} \mathbf{I}+\frac{1}{\Gamma} \mathbf{E}$, where $\hat{p_{s}}$ is the pressure in the solid layer, and $\mathbf{E}$ is the general nonlinear strain tensor given as $\mathbf{E}=\left(\mathbf{F} \cdot \mathbf{F}^{\mathbf{T}}-\mathbf{I}\right)$. Here, $\mathbf{F}=\nabla_{\mathbf{X} w}$ is the deformation gradient tensor and $\boldsymbol{w}(\boldsymbol{X})=\left(w_{X}, w_{Y}, w_{Z}\right)$ denotes the current position of the material particle in the deformed state of the solid layer. The parameter $\Gamma=\mu V / G R$, where $G$ is the shear modulus of the solid layer, is one of the most important parameter for problems under consideration characterizing the deformability of the solid layer. For rigid solids (with very high values of shear modulus $G \sim 10^{10} \mathrm{~Pa}$ ), $\Gamma \rightarrow 0$ and higher values of $\Gamma$ (equivalently lower shear modulus) indicate more and more softer solid. The dimensionless mass and momentum conservation equations for a neo-Hookean solid layer are

$$
\operatorname{det}(\mathbf{F})=1, \quad \operatorname{Re}\left[\frac{\partial^{2} \boldsymbol{w}}{\partial t^{2}}\right]_{\mathbf{X}}=\nabla_{\mathbf{X}} \cdot \mathbf{P}+\frac{\rho \mathbf{g} R^{2}}{\mu V}
$$

where $\mathbf{P}$ is the first Piola-Kirchhoff tensor and is related to Cauchy stress tensor as $\mathbf{P}=\mathbf{F}^{-1} \cdot \boldsymbol{\sigma}$.

For the falling film configuration shown in figure 1, the dimensionless base state velocity and pressure field in fluid layer are

$$
\bar{v}_{x}=\left(1-z^{2}\right), \quad \bar{v}_{z}=0, \quad \bar{p}=(2 \cot \theta) z .
$$




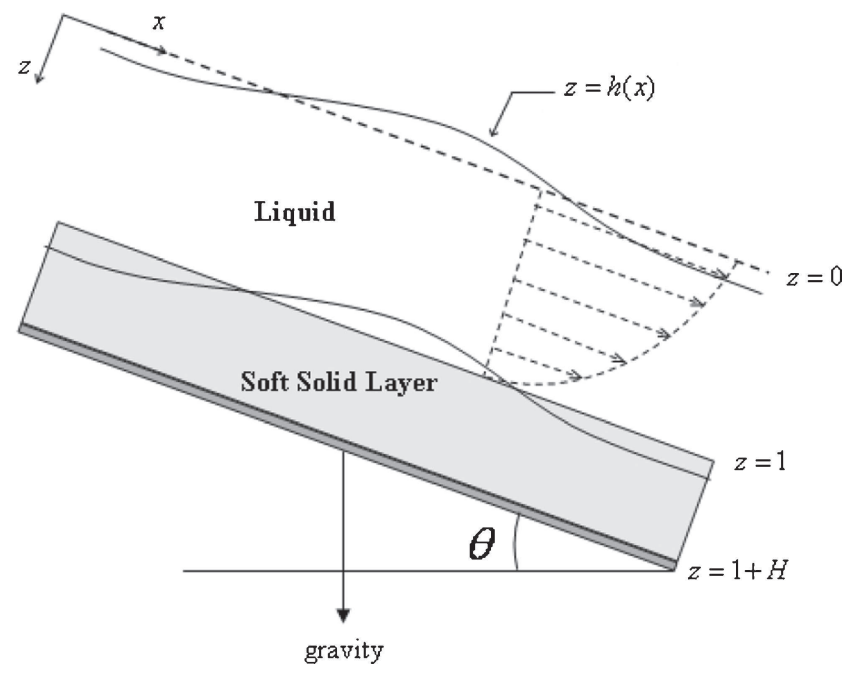

Figure 1. Schematic diagram showing the configuration and non-dimensional coordinate system: gravitydriven flow of a Newtonian liquid layer falling down an incline which is coated with a deformable solid layer.

and the base state deformation and pressure field in the solid layer are given as

$$
\bar{w}_{X}=X+\Gamma\left[(1+H)^{2}-Z^{2}\right], \quad \bar{w}_{Z}=Z, \quad \overline{\hat{p}}_{s}=\frac{1}{\Gamma}+(2 \cot \theta) Z .
$$

where $\hat{p}_{s}$ is a pressure-like function related to actual pressure in solid as $\hat{p}_{S}=p_{S}+\frac{1}{\Gamma}$.

A standard temporal linear stability analysis is carried out by imposing small perturbations on base state solution: $f=\bar{f}+f^{\prime}$. Here $f$ is any dynamical variable in liquid or solid layer, $\bar{f}$ denotes the base state and $f^{\prime}$ is the small perturbation imposed on $\bar{f}$. The perturbations are expanded in the form of normal modes: $f^{\prime}=\tilde{f}(z) \exp [\mathrm{i} k(x-c t)]$, where $\tilde{f}(z)$ is the complex amplitude function of the disturbance, $k$ is the wavenumber of perturbation and $c=c_{r}+\mathrm{i} c_{i}$ is the complex wavespeed. If $c_{i}>0$ (or $c_{i}<0$ ), flow will be unstable (or stable). For solid layer, $x$ and $z$ are replaced by $X$ and $Z$. Following the standard procedure, the governing equations for liquid layer are

$$
\begin{aligned}
\frac{\mathrm{d} \tilde{v}_{z}}{\mathrm{~d} z}+\mathrm{i} k \tilde{v}_{x} & =0, \\
\operatorname{Re}\left[\mathrm{i} k\left(\bar{v}_{x}-c\right) \tilde{v}_{x}+\left(\mathrm{d}_{z} \bar{v}_{x}\right) \tilde{v}_{z}\right] & =-\mathrm{i} k \tilde{p}+\left[\frac{\mathrm{d}^{2}}{\mathrm{~d} z^{2}}-k^{2}\right] \tilde{v}_{x}, \\
\operatorname{Re}\left[\mathrm{i} k\left(\bar{v}_{x}-c\right) \tilde{v}_{z}\right] & =-\frac{\mathrm{d} \tilde{p}}{\mathrm{~d} z}+\left[\frac{\mathrm{d}^{2}}{\mathrm{~d} z^{2}}-k^{2}\right] \tilde{v}_{z} .
\end{aligned}
$$

and for solid layer are

$$
\begin{aligned}
& \frac{\mathrm{d} \tilde{w}_{Z}}{\mathrm{~d} Z}+\mathrm{i} k \tilde{w}_{X}-\left(\frac{\mathrm{d} \bar{w}_{X}}{\mathrm{~d} Z}\right) \mathrm{i} k \tilde{w}_{Z}=0, \\
&-\mathrm{i} k \tilde{p}_{s}+\underline{(2 \cot \theta) \mathrm{i} k \tilde{w}_{Z}}+\frac{1}{\Gamma}\left[-k^{2}+\frac{\mathrm{d}^{2}}{\mathrm{~d} Z^{2}}\right] \tilde{w}_{X}=k^{2} c^{2} \operatorname{Re} \tilde{w}_{X}, \\
&-\underline{(2 \cot \theta) \mathrm{i} k \tilde{w}_{X}}-\frac{\mathrm{d} \tilde{p}_{s}}{\mathrm{~d} Z}+\frac{1}{\Gamma}\left[-k^{2}+\frac{\mathrm{d}^{2}}{\mathrm{~d} Z^{2}}\right] \tilde{w}_{Z}+\underline{\left(\frac{\mathrm{d} \bar{w}_{X}}{\mathrm{~d} Z}\right) \mathrm{i} k \tilde{p}_{S}}=k^{2} c^{2} \operatorname{Re} \tilde{w}_{Z} .
\end{aligned}
$$


The linearized kinematic and boundary conditions at unperturbed free surface $(z=0)$ are obtained by Taylor-expanding the conditions about $z=0$ :

$$
\begin{aligned}
\mathrm{i} k\left[\bar{v}_{x}(z=0)-c\right] \tilde{h} & =\tilde{v}_{z}(z=0), \\
-2 \tilde{h}+\left(\frac{\mathrm{d} \tilde{v}_{x}}{\mathrm{~d} z}+\mathrm{i} k \tilde{v}_{z}\right) & =0, \\
-\tilde{p}-(2 \cot \theta) \tilde{h}+2 \frac{\mathrm{d} \tilde{v}_{z}}{\mathrm{~d} z}-k^{2} \Sigma_{g l} \tilde{h} & =0 .
\end{aligned}
$$

where $\Sigma_{g l}=\gamma_{g l} / \mu V$ is the nondimensional GL surface tension with $\gamma_{g l}$ being the dimensional surface tension. The linearized velocity and stress continuity conditions at LS interface are

$$
\begin{aligned}
\tilde{v}_{z} & =-\mathrm{i} k c \tilde{w}_{Z}, \\
\left.\left(\frac{\mathrm{d} \tilde{w}_{X}}{\mathrm{~d} Z}+\mathrm{i} k \tilde{w}_{Z}\right)+\left(\frac{\mathrm{d} \bar{w}_{X}}{\mathrm{~d} Z}\right) \frac{\mathrm{d} \tilde{w}_{Z}}{\mathrm{~d} Z}-\left(\frac{\mathrm{d} \bar{w}_{X}}{\mathrm{~d} Z}\right)^{2} \mathrm{~d} \mathrm{~d}_{z} \bar{v}_{x}\right)_{z}=1 & =\Gamma\left(\frac{\mathrm{d} \tilde{w}_{X}}{\mathrm{~d} z}+\mathrm{i} k \tilde{v}_{z}\right)+\tilde{w}_{Z}\left(\mathrm{~d}_{z}^{2} \bar{v}_{x}\right) \Gamma, \\
-\tilde{p}_{s}+\frac{2}{\Gamma} \frac{\mathrm{d} \tilde{w}_{Z}}{\mathrm{~d} Z}-k^{2} \Sigma_{l s} \tilde{w}_{Z}+\tilde{w}_{Z} \frac{\mathrm{d} \bar{p}}{\mathrm{~d} z} & =-\tilde{p}+2 \frac{\mathrm{d} \tilde{v}_{z}}{\mathrm{~d} z}
\end{aligned}
$$

Finally, the boundary conditions at rigid surface $(z=1+H)$ are zero displacement conditions $\tilde{\boldsymbol{w}}=0$. Equations (4)-(18) govern the linear stability of the falling film configuration shown in figure 1. A similar set of equations governing the linear stability can be derived for other flow configurations.

\section{Effect of solid layer on long-wave interfacial instabilities}

The jump in viscosity/elasticity across a fluid-fluid interface results in a long wavelength instability; therefore, we first discuss the effect of deformable solid layer on long wavelength disturbances. In the following, we use falling film configuration shown in figure 1 as a representative example to briefly discuss the salient features of long-wave asymptotic analysis. Subsequently, we discuss long-wave results for other flow configurations and for different fluid rheological behaviors.

\subsection{Suppression of gas-liquid free surface instability}

For solid thickness $H \sim O(1), k \ll 1$ implies long-wave or low wavenumber limit. The complex wavespeed $c$ is expanded in an asymptotic series in $k: c=c^{(0)}+k c^{(1)}+\cdots$. The variables in fluid and solid layers are also expanded in asymptotic series in $k$. For example, if we set $\tilde{v}_{z} \sim O(1)$, then Eqs. 6 and 7 respectively, imply $\tilde{v}_{x} \sim O\left(k^{-1}\right)$ and $\tilde{p} \sim O\left(k^{-2}\right)$. Thus, $\tilde{v}_{z}=\left(\tilde{v}_{z}^{(0)}+k \tilde{v}_{z}^{(1)}+\cdots\right), \tilde{v}_{x}=k^{-1}\left(\tilde{v}_{x}^{(0)}+k \tilde{v}_{x}^{(1)}+\cdots\right), \tilde{p}=k^{-2}\left(\tilde{p}^{(0)}+k \tilde{p}^{(1)}+\cdots\right)$, and similarly for variables in solid layer. Such expansions for both fluid and solid layers are substituted in governing stability equations (Eqs. 6-8 and 9-11) and the velocity field in fluid layer and deformation field in solid layer are obtained at each order of $k$ analytically. The velocity in fluid and deformations in solid are coupled via interfacial conditions at LS interface. On substitution of expansions in velocity and stress continuity conditions at LS interface, it turns out that the leading order velocity field satisfies no slip conditions $\left(\tilde{v}_{z}^{(0)}=0, \tilde{v}_{x}^{(0)}=0\right)$ similar to the case of flow past rigid surface. Further, the equations at leading order show that the fluid can be solved independent of solid and the leading order velocity field in the presence of soft solid 
layer remains identical to leading order velocity field for flow past rigid incline. Therefore, the leading order wavespeed $c^{(0)}$ remains identical to the case of flow of falling film down a rigid incline. This conclusion of $c^{(0)}$ being identical to the rigid counterpart holds for any composite fluid-solid flow configuration involving at least one LL/GL interface. The value is determined to be $c^{(0)}=2$ for falling film configuration. Thus, the configuration is neutrally stable at this order. However, liquid layer exerts tangential stress at LS interface $\left(\mathrm{d}_{z} \tilde{v}_{x}^{(0)}=1 / \Gamma \mathrm{d}_{z} \tilde{w}_{X}^{(0)}\right)$ which imparts a nonzero displacement in the solid layer at leading order. This leading order deformation field set-up in solid layer by leading order velocity field affects the first correction to velocity field as evident from velocity continuity conditions at $O(k)$.

$$
\tilde{v}_{z}^{(1)}=-\mathrm{i} c^{(0)} \tilde{w}_{Z}^{(0)}, \quad \tilde{v}_{x}^{(1)}+\left.\mathrm{d}_{z} \bar{v}_{x}\right|_{z=1} \tilde{w}_{Z}^{(0)}=-\mathrm{i} c^{(0)} \tilde{w}_{X}^{(0)} .
$$

Thus, the leading order deformation field could affect the first correction to wavespeed. Subsequent calculations for $c^{(1)}$ indeed confirm this and the first correction to wavespeed is found out to be purely imaginary and hence, determine the stability of composite fluid-solid system in the low wavenumber limit.

$$
c^{(1)}=\frac{2 \mathrm{i}}{15}[(\underline{4 R e-5 \cot \theta})-30 \Gamma H] .
$$

The above expression for $c^{(1)}$ consists of two qualitatively different contributions. First, the underlined terms, proportional to $R e$ and $\cot \theta$, are exactly identical to the expression for $c^{(1)}$ for flow past rigid incline (Yih 1963) and hence, the underlined terms are referred as rigid contribution. Second, the boxed term proportional to solid layer deformability $(\Gamma)$ and thickness of solid layer $(H)$ is referred as soft solid contribution. For other flow configurations as well, the expression for $c^{(1)}$ always consists of a rigid contribution and a soft solid contribution with rigid contribution remaining exactly identical to the expression for $c^{(1)}$ as obtained if no deformable layer is present. The above equation for $c^{(1)}$ clearly shows that the falling film becomes unstable to low- $k$ disturbances when $R e>5 / 4 \cot \theta$ in the limit of rigid solid $(\Gamma \rightarrow 0)$ or in the absence of deformable solid layer $(H \rightarrow 0)$. The term proportional to $\Gamma$ and $H$ occurs with negative sign which implies that the soft solid contribution is always stabilizing on GL interfacial mode. Thus, a soft solid coating (i.e. nonzero $H$ ) with appropriately chosen shear modulus (i.e. deformability parameter $\Gamma$ ) is capable of suppressing free surface instability in long-wave limit when it was otherwise unstable for a rigid incline.

If the falling film is a viscoelastic liquid modeled using an Oldroyd-B constitutive relation, then the expressions for $c^{(0)}$ and $c^{(1)}$ are given as

$$
c^{(0)}=2 ; \quad c^{(1)}=\frac{2 \mathrm{i}}{15}[(4 R e-5 \cot \theta)+10 W(1-S)-30 \Gamma H] .
$$

Again, the rigid contribution is marked by an underline while the soft solid contribution is represented by boxed term. The rigid contribution contains terms proportional to $R e$ and $\cot \theta$ which remain present for a Newtonian film and an additional destabilizing term proportional to Weissenberg number $W$ representing the elastic nature of liquid film. The leading order wavespeed is also identical to the Newtonian case $\left(c^{(0)}=2\right)$. More interestingly, the soft solid contribution also remains identical to the case of Newtonian film indicating that it is independent of the fluid rheological behavior. The details of low- $k$ analysis reveal that the leading order velocity field developed in the liquid layer, even on using an Oldroyd-B model, matches exactly with the 
leading order velocity field developed for Newtonian film. The low- $k$ analysis presented above shows that the leading order velocity field is responsible for setting of leading order deformation field in solid layer which in turn affects the first correction to wavespeed $c^{(1)}$. Since the leading order velocity and deformation field remain identical to the Newtonian case, the soft solid contribution in above equation matches perfectly with that given in Eq. (20) for Newtonian falling film. Thus, it is possible to use a deformable solid coating to suppress the free surface instability even for a viscoelastic liquid film.

\subsection{Simultaneous suppression/manipulation of GL and LL interface}

We next consider the gravity driven flow of two Newtonian liquid layers falling down an incline (refer figure 2) to explore the possibility of simultaneous control of both GL and LL interfacial instabilities by using a deformable solid coating. Here, $\beta$ is the dimensionless thickness of top liquid (Liquid A) layer and $\mu_{\mathrm{r}}=\mu_{a} / \mu_{b}$ is the viscosity ratio of top and bottom liquid layers. In the absence of deformable solid layer, i.e. in the rigid limit, the stability behavior depends crucially on whether less viscous liquid is adjacent to wall $\left(\mu_{\mathrm{r}}>1\right)$ or more viscous liquid is near the inclined surface $\left(\mu_{\mathrm{r}}<1\right)$. We first discuss the case of more viscous fluid near the wall and as a representative example, we fix $\mu_{\mathrm{r}}=0.5$ and $\beta=0.5$. Here, we obtain two values of $c^{(0)}$ and $c^{(1)}$ corresponding to two interfacial (GL and LL) modes.

$$
\begin{aligned}
& c_{g l}^{(0)}=1.80902, \quad c_{g l}^{(1)}=\mathrm{i}[\underline{0.904 R e-0.720 \cot \theta}-4.319 \Gamma H], \\
& c_{l l}^{(0)}=0.82461, \quad c_{l l}^{(1)}=\mathrm{i} \times 10^{-2}[\underline{0.386 R e-3.011 \cot \theta}-18.07 \Gamma H] .
\end{aligned}
$$

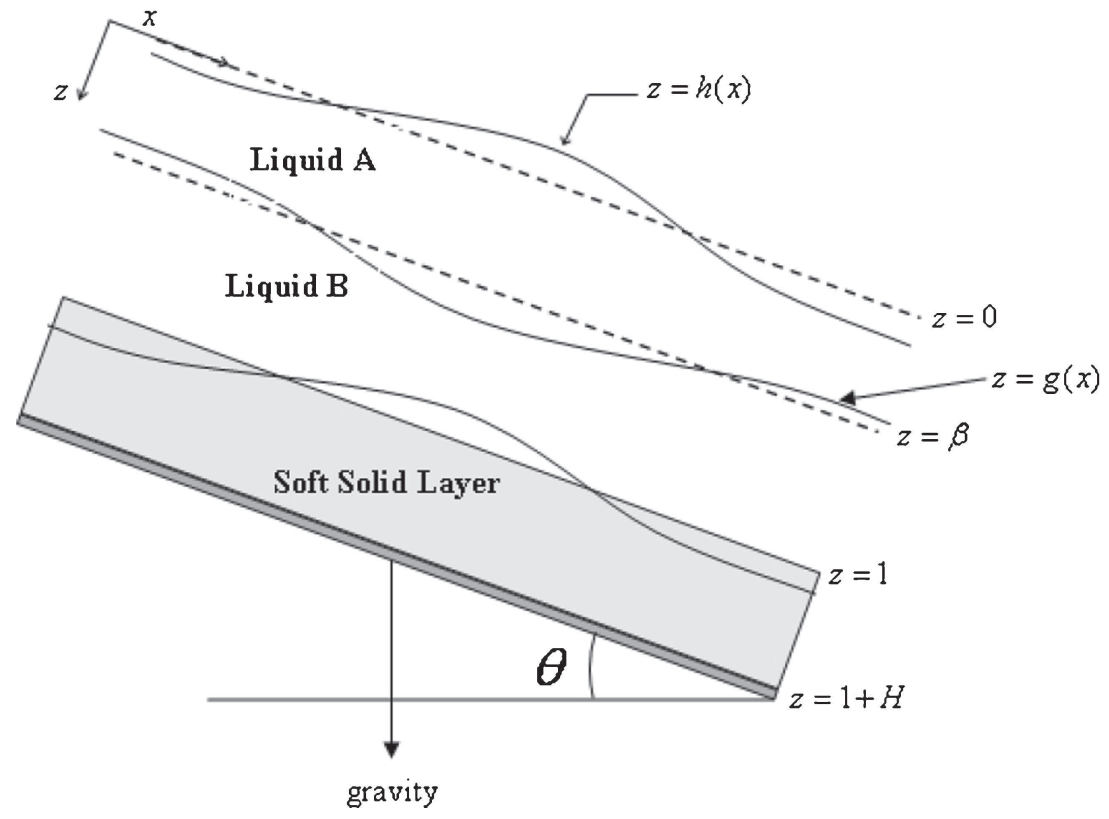

Figure 2. Schematic diagram showing the configuration and (non-dimensional) coordinate system: two Newtonian liquid films flowing past an inclined plane lined with an elastic neo-Hookean solid layer. 
For both GL and LL interfacial modes, the term proportional to $R e$ is destabilizing and the term proportional to $\cot \theta$ is stabilizing. Thus, both GL and LL interfaces can become unstable as $R e$ increases above a critical value in the absence of deformable solid layer $(\Gamma$ or $H=0)$. The

expressions for $c_{g l}^{(1)}$ and $c_{l l}^{(1)}$ show that the soft solid contribution is stabilizing for both interfaces and hence it is possible to appropriately choose the values of $\Gamma$ and $H$ such that both GL and LL mode perturbations can be suppressed. We have verified that such suppression of both GL and LL interfaces holds for other values of $\beta$ as well for $\mu_{\mathrm{r}}<1$.

We fix $\mu_{\mathrm{r}}=2$ and $\beta=0.5$ for discussing $\mu_{\mathrm{r}}>1$ case. The low- $k$ results for both interfacial modes are

$$
\begin{gathered}
c_{g l}^{(0)}=1.80902, \quad c_{g l}^{(1)}=\mathrm{i}[\underline{0.406 R e-0.638 \cot \theta}-\underline{3.831 \Gamma H}], \\
c_{l l}^{(0)}=0.69098, \quad c_{l l}^{(1)}=\mathrm{i} \times 10^{-2}[\underline{-0.120 R e+1.36 \cot \theta}+8.156 \Gamma H]
\end{gathered}
$$

The expression for $c_{g l}^{(1)}$ shows that the GL mode perturbations can still be suppressed by suitably choosing values of $\Gamma$ and $H$ in a manner similar to $\mu_{\mathrm{r}}<1$. However, the qualitative nature of both rigid and soft solid contributions for $c_{l l}^{(1)}$ is reversed as compared to the $\mu_{\mathrm{r}}<1$ case. The term proportional to $R e$ becomes stabilizing while the terms proportional to $\cot \theta$ and $\Gamma H$ are destabilizing. Thus, the deformable solid layer has stabilizing effect on GL interface but destabilizing effect on LL interface. For given $R e$ and $\theta$, the above expression for $c_{l l}^{(1)}$ suggests that $(\Gamma H)<(0.0147 R e-0.167 \cot \theta)$ for LL interface to remain stable. However, Eq. (24) shows that $(\Gamma H)>(0.106 R e-0.167 \cot \theta)$ in order to stabilize GL interface. Thus, it is not possible to simultaneously suppress both GL and LL mode perturbations by using a soft solid coating for $\mu_{\mathrm{r}}=2$ and $\beta=0.5$. We have verified this conclusion for other values of $\beta$ and $\mu_{\mathrm{r}}>1$. Interestingly, the above discussion indicates selective destabilization of LL interface by wall deformability for $\mu_{\mathrm{r}}>1$. The values of $\Gamma$ and $H$ can be selected in such a way that the GL interface perturbations are suppressed while the LL interface becomes unstable. Such selective destabilization of one interface can be of potential use in pattern transfer applications or in generation of micro-droplets in microfluidic devices which utilize interfacial instabilities to produce drops or particles of desired size. For the sake of brevity, we do not present the results for the control of LL interfacial instabilities in channel flows and simply remark here that a similar manipulation and control of LL interfacial instability is observed in these cases as well.

\subsection{Simultaneous suppression of capillary and interfacial instabilities}

All the results presented above correspond to planar flow configurations for which surface tension forces remain sub-dominant in the long-wave limit. Here, we consider pressure-driven core-annular flow arrangement of two immiscible Newtonian liquids in a flexible tube where, due to the presence of cylindrical fluid-fluid interface, capillary instability is also present in addition to fluid-fluid interfacial instabilities caused due to viscosity contrast across the interface. The schematic is shown in figure 3. Preziosi et al (1989) have shown that it is possible to obtain stable core-annular flow (CAF) in a rigid tube when the less viscous fluid is adjacent to the wall (i.e. $\mu_{\mathrm{r}}=\mu_{a} / \mu_{b}>1$ ) and occupies sufficiently less space than the more viscous core fluid. The other configuration when more viscous fluid is near the wall $\left(\mu_{\mathrm{r}}<1\right)$ is always unstable. We 


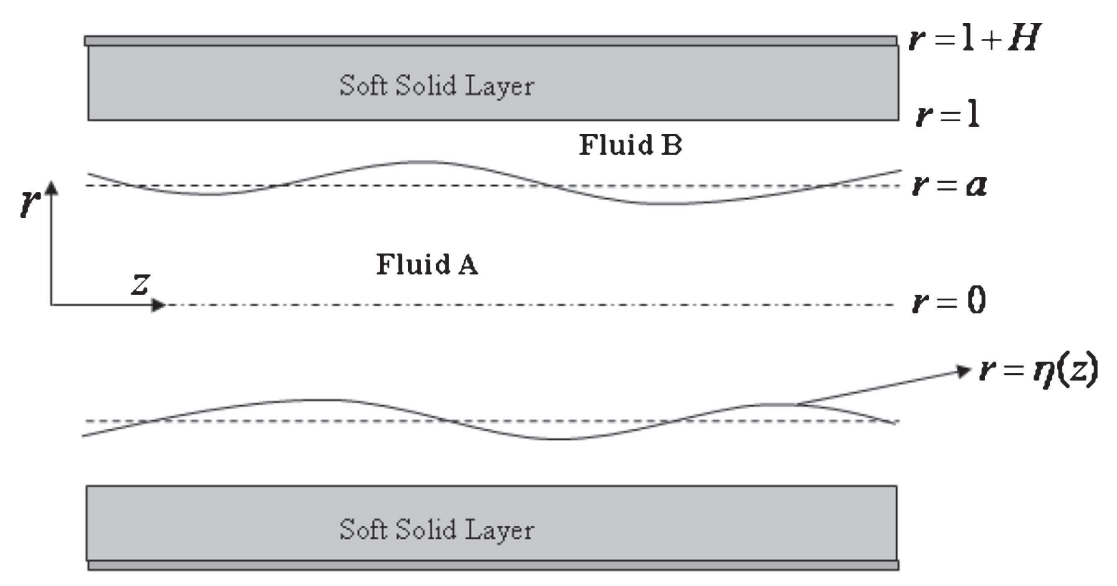

Figure 3. Schematic diagram showing the configuration and (non-dimensional) coordinate system: two immiscible fluids flowing in core-annular arrangement in a tube coated with deformable solid layer.

first discuss $\mu_{\mathrm{r}}<1$ case and as a representative example, we set $\mu_{\mathrm{r}}=0.5, a=0.7$, and $H=2$. The leading order and first correction to wavespeed are

$$
\begin{aligned}
c & =c^{(0)}+k c^{(1)} \\
& =0.41125+k \mathrm{i}\left(\underline{\left.4.1462 \times 10^{-6} R e+8.8005 \times 10^{-3} \Sigma-2.61970 \Gamma\right)}\right) .
\end{aligned}
$$

The above expression clearly shows that the term proportional to $R e$, present due to viscosity stratification, and the term proportional to dimensionless interfacial tension $\Sigma$ (capillary instability) are both destabilizing and hence CAF remains unstable in rigid limit $(\Gamma=0)$. The soft solid contribution (boxed term) is stabilizing and thus deformability parameter $\Gamma$ can be appropriately chosen to suppress both capillary and two-fluid interfacial instabilities. The above equation is written for $\mu_{\mathrm{r}}=0.5$ and $a=0.7$; but, we have verified that the nature of soft solid contribution is stabilizing for all values of $a$ and $\mu_{\mathrm{r}}<1$. A similar suppression of capillary and GL free surface instabilities was also observed for the case of liquid film flow outside/inside of a tube coated with deformable solid layer (Jain \& Shankar 2008). The nature of rigid and soft solid contributions in the expression of $c^{(1)}$ for CAF changes when less viscous fluid occupies the annular space. If $\mu_{\mathrm{r}}=2, a=0.7$, and $H=2, c^{(0)}$ and $c^{(1)}$ are given as

$$
c=0.57957+k \mathrm{i}\left(\underline{\left.-4.1336 \times 10^{-5} \operatorname{Re}+4.7319 \times 10^{-3} \Sigma 3.66622 \Gamma\right)} .\right.
$$

Note that among the rigid contributions, the term proportional to interfacial tension is still destabilizing while the contribution due to viscosity stratification has now become stabilizing and it is this term which is responsible for stabilizing CAF system in rigid limit. However, the presence of deformable solid layer is destabilizing for this case and hence, the stable CAF can be rendered unstable by varying the wall deformability parameter $\Gamma$. We have verified that this destabilizing nature of wall elasticity is present for different values of mean LL interface position and $\mu_{\mathrm{r}}>1$. The above discussion clearly shows that the soft solid layer could have a stabilizing or destabilizing effect depending on whether more or less viscous fluid is present near the wall. 


\section{Numerical results: manipulation for arbitrary wavelength disturbances}

The results in Section 3 indicated the potential of using a deformable solid layer in suppressing or enhancing the fluid-fluid interfacial instabilities, but, are restricted to long wavelength disturbances. In this section, we extend the above low- $k$ results to arbitrary wavenumbers to ensure whether the predicted suppression in long wave limit holds for finite and high wavenumber perturbations as well. Furthermore, flow past deformable solid surface (involving only a fluid-solid interface) could become unstable on increasing the deformability in the absence of inertia (Kumaran et al 1994; Gkanis \& Kumar 2003), and several additional unstable fluid-solid modes proliferate when inertia is present (Chokshi \& Kumaran 2008; Gaurav \& Shankar 2009, 2010b). All these fluid-solid unstable modes are not captured by the low- $k$ analysis presented in the previous section. For stable flow configuration, fluid-solid interface must also remain stable for parameter regime where solid layer deformability has stabilizing effect on fluid-fluid interfacial mode. We used a spectral collocation method which resolves the complete eigenspectrum at any arbitrary wavenumber and hence captures the complex wavespeeds corresponding to all interfaces present in the system. The composite fluid-solid system usually involves one unstable eigenvalue corresponding to each fluid-fluid interface present in the system and multiple unstable eigenmodes corresponding to fluid-solid interface. The identification of fluid-fluid (GL or LL) interfacial mode is done by comparing the eigenspectrum obtained from spectral collocation method at low- $k$ values with the long-wave asymptotic results for a particular GL/LL interface. The eigenvalue which matches with the complex wavespeed predicted from low- $k$ analysis is identified as GL/LL interfacial mode. Any other unstable mode is referred as fluid-solid or LS interfacial mode.

\subsection{Suppression of interfacial instabilities}

Most of the results are presented in the form of $\Gamma$ vs $k$ neutral curves demarcating stable and unstable regions for a given set of parameters. We do not provide extensive data, but, present key results for different flow configurations demonstrating the main aspect that the solid layer deformability parameter could be adjusted so as to suppress or enhance fluid-fluid interfacial instabilities. Figure 4 shows neutral stability diagram for gravity-driven flow of an OldroydB viscoelastic liquid film falling down a vertical incline coated with deformable solid layer. The expression of $c^{(1)}$ (see Eq. 21) shows that the viscoelastic liquid film falling down a vertical incline becomes unstable for any nonzero $R e$ and $W$. Thus, in figure 4 for $R e=0.1$ and $W=0.5$, the free surface remains unstable in rigid limit $\Gamma \rightarrow 0$. As deformability parameter $\Gamma$ is gradually increased above the lower neutral curve, the GL free surface instability is suppressed and we obtain a stable region where both GL and LS interfaces remain unperturbed. The lower neutral curve is obtained by extending the long-wave asymptotic results (Eq. 21) to finite wavenumbers and shows that the suppression predicted in low- $k$ limit holds for any arbitrary wavenumber. With further increase in $\Gamma$, we obtain several neutral curves corresponding to destabilization of GL mode and multiple LS modes. There exists a wide gap in terms of deformability parameter $\Gamma$ where both interfaces remain stable and hence, the viscoelastic film which was unstable in rigid limit becomes stable in pressure of a deformable solid coating. We have verified the existence of such a wide stable gap for a wide variety of parameters, for example, for Newtonian films (i.e. $W=0$ ) at different values of angle of inclinations. The above result was presented for $W=0.5$ and it is well known that fluid elasticity has destabilizing effect on fluidfluid interfacial mode. We observed that the width of stability window decreases with increase in Weissenberg number and it finally vanishes when $W$ increases above a critical value (results 


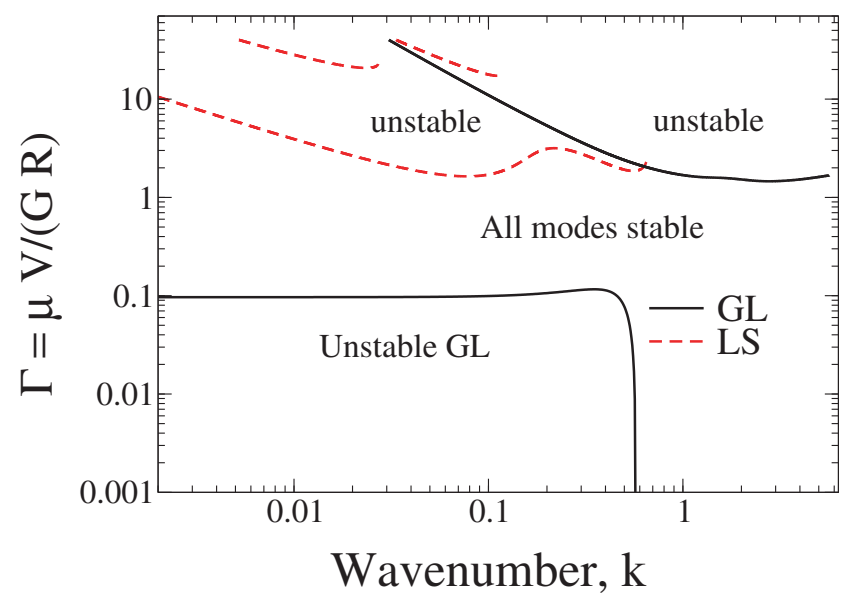

Figure 4. Suppression of free surface instability for a viscoelastic film falling down an incline: $\Gamma$ vs $k$ for $R e=0.1, W=0.5, S=0.5, \theta=90^{\circ}, \Sigma_{g l}=2, \Sigma_{l s}=1$ and $H=1$.

not shown). A similar closing of stability window is observed in case of Newtonian falling film as well when $R e$ is sufficiently increased. Thus, the conclusion of free surface instability suppression holds for both Newtonian and viscoelastic liquid films for a range of values of $R e$ and $W$ when the film was otherwise unstable in rigid limit. However, when $R e$ and $W$ are large, it is not possible to obtain stable flow configuration.

We next consider gravity-driven flow of two Newtonian liquid layers falling down an incline surface in order to examine the possibility of simultaneous suppression of GL and LL interfacial modes in the presence of a deformable solid coating. The low- $k$ results demonstrated that the role of deformable solid coating is stabilizing for $\mu_{\mathrm{r}}<1$ and destabilizing for $\mu_{\mathrm{r}}>1$. Here, we present the neutral curves for the case when the soft solid contribution is stabilizing. Figure 5 depicts neutral stability diagrams for $\mu_{\mathrm{r}}=0.5, \beta=0.5$ and $R e=0.1$ for a vertical incline. The GL and LL interfaces remain unstable for flow down a rigid vertical incline for above given parameters (for example, refer Eqs. (22) and (23) for $\Gamma=0$ or $H=0$ ). As $\Gamma$ is increased, the LL interface becomes stable and with further increase in $\Gamma$ above lower GL mode neutral curve, the GL interface also becomes stable. Following this, there exist a range of $\Gamma$ values where both GL and LL modes are stabilized due to solid deformability while the LS interface remains unperturbed. Thus, the two-liquid film flow becomes stable at $R e=0.1$ for a range of values of solid deformability parameter. When $\Gamma$ is sufficiently increased, all three interfaces become unstable as shown by the presence of three upper neutral curves for each of GL, LL and LS interfaces. The existence of stability window depends strongly on mean dimensionless top liquid layer thickness $\beta$. The neutral stability curves for different values of $\beta$ indicate that increasing $\beta$ reduces the width of stable gap and we observed that for $\beta=0.7$, the width of stable gap decreases significantly (results not shown here) so as to close the gap for all practical purposes.

We now consider core-annular flow of two immiscible Newtonian liquids in a flexible tube for which the fluid-fluid interface becomes unstable due to both capillary forces and viscosity stratification in the rigid limit. The asymptotic analysis presented above demonstrated that the low- $k$ perturbations can be suppressed by using a deformable solid coating for $\mu_{\mathrm{r}}<1$ (see Eq. 26). Figure 6 shows results in terms of $\Gamma$ vs $k$ neutral curves for all wavenumbers for $\mu_{\mathrm{r}}=$ 0.5 , mean interface position $a=0.9$, and at two different values of Reynolds number. For 


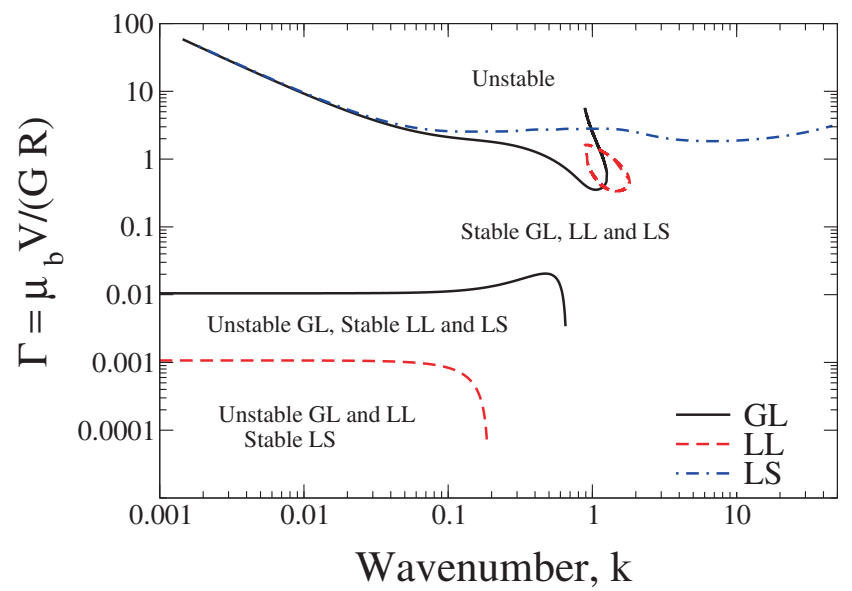

Figure 5. Simultaneous suppression of free surface and liquid-liquid interfacial instabilities for two-layer Newtonian film flow down an incline in the presence of a deformable solid coating: $\Gamma$ vs $k$ for $\mu_{\mathrm{r}}=$ $0.5, \beta=0.5, \operatorname{Re}=0.1,, \theta=90^{\circ}, \Sigma_{g l}=\Sigma_{l l}=0.25, \Sigma_{l s}=0$ and $H=2$.

$R e=0.1$ in figure 6, the fluid-fluid interface remains unstable due to nonzero $\Sigma_{l l}$ and $R e$ in the limit $\Gamma \rightarrow 0$. The neutral curves presented in figure 6 clearly show the existence of a wide stable gap in terms of deformability parameter $\Gamma$ where the flow configuration is stabilized in the presence of sufficiently soft solid coating. When $R e$ is increased to 5, the lower LL mode neutral curve, which gives transition value of $\Gamma$ above which flow becomes stable, significantly shifts upwards. On the other hand, the critical $\Gamma$ value above which the LL and LS interfaces become unstable (shown by upper neutral curves) does not alter significantly. As a result of this, the stability window shrinks significantly and is quite small as compared to $R e=0.1$. A parametric investigation with respect to variation of $R e$ shows that the stable gap vanishes for $R e \sim O(10)$, and that stable CAF arrangement in deformable tube could be obtained only for $R e \lesssim O(1)$.

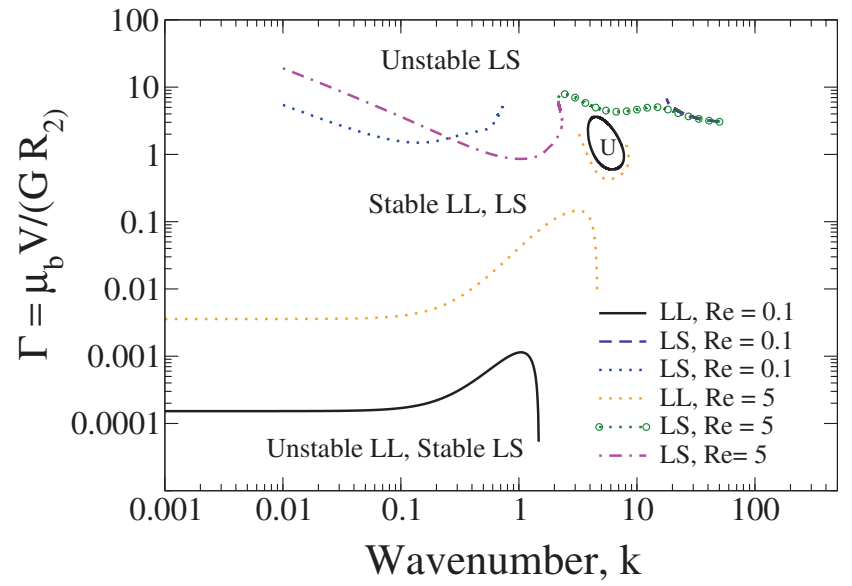

Figure 6. Suppression of capillary and liquid-liquid interfacial instabilities for CAF in a flexible tube: $\Gamma$ vs $k$ for $\mu_{\mathrm{r}}=0.5, a=0.9, \Sigma_{l l}=0.1, \Sigma_{l s}=0$ and $H=2$. 


\subsection{Selective destabilization or enhancement of interfacial instabilities}

The low- $k$ results for Newtonian/viscoelastic single liquid falling film with a free surface, in either planar or cylindrical geometry, show that the soft solid contribution is always stabilizing for GL free surface. Thus, the only possibility of GL interface becoming unstable due to solid deformability is depicted by upper neutral curves presented in the previous section (for example, see figure 4). However, for flow configurations involving two fluid layers, the soft solid has a destabilizing effect depending on $\mu_{\mathrm{r}}$ in low- $k$ limit, and solid deformability could be altered to render flow unstable for those values of parameters where the flow otherwise remains stable in rigid limit. In this section, we examine such cases to investigate the role of deformable solid layer in enhancing or selectively destabilizing the fluid-fluid interfacial instabilities. Figure 7 depicts neutral curves for two Newtonian liquid films falling down a vertical incline coated with deformable solid layer. The data is presented for $\mu_{\mathrm{r}}=2, \beta=0.5$ and $H=2$. The expression of $c^{(1)}$ for GL and LL interfaces in Eqs. (24) and (25), respectively for $\mu_{\mathrm{r}}=2$ and $\beta=0.5$ clearly shows that the solid layer has stabilizing effect on GL interface, but have a destabilizing effect on LL interface perturbations. In rigid limit $(\Gamma \rightarrow 0)$ for $\theta=90^{\circ}, R e=0.1$, the GL interface remains unstable while the LL and LS interfaces are stable. As $\Gamma$ is increased over lower LL mode neutral curve, there is a transition from stable to unstable perturbation for LL interface due to increase in solid layer deformability. The LL mode neutral curve is now a single curve with region left to it being unstable and region right to it being stable. The GL and LS interfaces become unstable on sufficiently increasing deformability parameter $\Gamma$ as depicted by presence of upper GL and LS neutral curves. For region in between lower and upper GL mode neutral curves, the LL interface is selectively destabilized due to solid layer deformability while GL mode perturbations are suppressed by soft solid layer without exciting LS mode perturbations.

We observed a similar destabilization of LL interface for the case of core-annular flow of two immiscible Newtonian liquids in a flexible tube (results not shown) when less viscous liquid is adjacent to the wall (i.e. $\mu_{\mathrm{r}}>1$ ). Recall that the low- $k$ result presented in Eq. (27) shows that the destabilizing contribution comes from term proportional to interfacial tension $\Sigma_{l l}$ and soft solid term which is proportional to $\Gamma$. The term proportional to $R e$ is stabilizing. We set

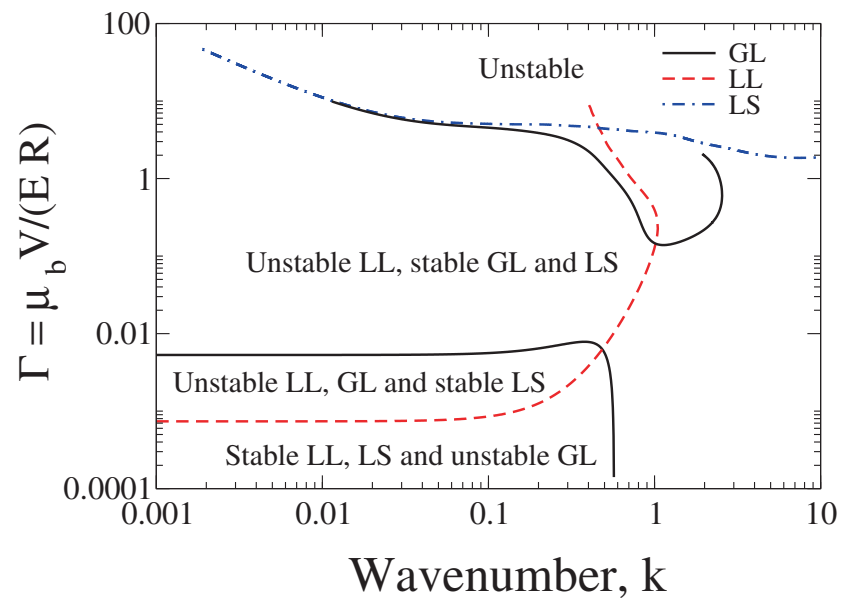

Figure 7. Neutral curves showing selective destabilization of LL interface for flow of two liquid films down an incline plane coated with deformable solid layer: $\Gamma$ vs $k$ for $\mu_{\mathrm{r}}=2, \beta=0.5, R e=0.1 \Sigma_{l l}=$ $\Sigma_{g l}=0.1, \Sigma_{l s}=0, \theta=90^{\circ}$ and $H=2$. 


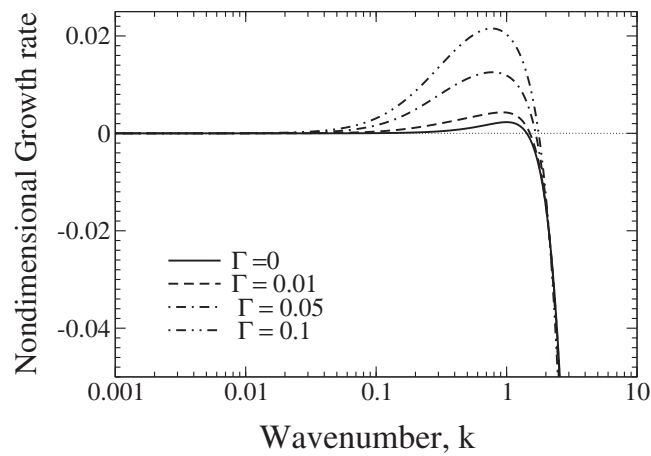

Figure 8. Growth rate vs wavenumber curves demonstrating enhancement of rate of growth of perturbations on increasing deformability: Data for $\mu_{\mathrm{r}}=2, a=0.7, R e=0, \Sigma_{l l}=1, \Sigma_{l s}=0$, and $H=2$.

$R e=0$ to suppress any stabilizing effect and compare the two destabilizing contributions present in Eq. (27). Figure 8 shows the growth rate vs wavenumber data for $H=2, R e=0$ and $\Sigma_{l l}=1$ at different values of $\Gamma$. This figure clearly shows that the rate of growth of perturbations can be significantly enhanced by making walls more and more deformable. For example, the maximum growth rate with $\Gamma=0.1$ is at least an order of magnitude higher than the growth rate $(\sim O(0.001))$ for $\Gamma=0$. This feature could be potentially used in increasing mixing rates of two fluids in microchannels which are often fabricated using soft elastomers.

We finally present neutral curves for $\mu_{\mathrm{r}}=0.5, a=0.3, \Sigma_{l l}=0.1$ and $H=2$ for CAF in deformable tube in figure 9. For $\mu_{\mathrm{r}}<1$, the effect of soft solid layer is stabilizing for twofluid interfacial mode in long wave limit. Figure 9 shows that the fluid-fluid interface remains unstable for $\Gamma \rightarrow 0$ due to capillary instability. The interface becomes stable for perturbations with wavenumbers $k<1$ as $\Gamma$ increases above a threshold value, However, for $k \approx 1-3$, the strong destabilizing capillary forces dominate over stabilizing soft solid contribution and the flow remains unstable within this band of wavenumbers. Thus, while stabilization of LL

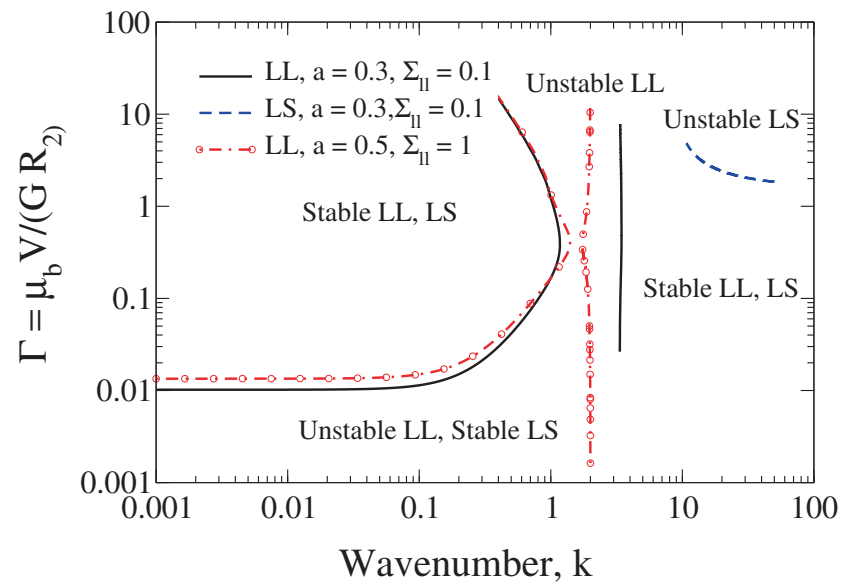

Figure 9. Neutral curves showing selective destabilization for CAF in deformable tube: Data for $\mu_{\mathrm{r}}=$ $0.5, R e=0, \Sigma_{l s}=0$, and $H=2$. 
interface is not achieved for this parameter set; but, this figure shows that a selective band of wavelengths can be excited by tuning the wall properties and interfacial tension. Figure 9 also shows data for $\mu_{\mathrm{r}}=0.5, a=0.5, \Sigma_{l l}=1$ and $H=2$. This set of data shows that the band of unstable wavenumbers become more selective for $a=0.5$ and $\Sigma=1$. Such selective destabilization of a band of particular wavelength perturbations could be utilized in producing mono-dispersed microdroplets in microchannels which relies on instability of a fluid-fluid interface for generating micro-sized drops.

\section{Conclusions}

In the present paper, we discussed the role of deformable solid coatings on liquid-liquid and gas-liquid interfacial instabilities for a variety of flow settings and for both Newtonian and viscoelastic fluids. The low wavenumber asymptotic analysis revealed that the soft solid layer can have a stabilizing effect on fluid-fluid interfacial mode when the interface remains unstable in the rigid limit. Numerical results demonstrated that the stabilizing effect of deformable solid layer continues to finite and high wavenumbers as well. The neutral curves clearly illustrated the presence of a sufficiently wide range of solid deformability parameter $\Gamma$ (or alternatively shear modulus of solid layer) where interfacial instabilities are completely suppressed at all wavenumbers. Further, the suppression was shown to be generic in nature and holds for both Newtonian and viscoelastic liquid layers. The results also demonstrated that it is possible to destabilize the interface by using a soft solid coating when it is stable in the absence of deformable solid coating. Thus, the present work demonstrates the efficacy of deformable solid coatings in manipulation and control of interfacial instabilities.

\section{References}

Bassom A P, Blyth M G and Papageorgiou D T 2012 Using surfactants to stabilize two-phase pipe flows of coreannular type. J. Fluid Mech. 704: 333-359

Chen K 1991 Interfacial instability due to elastic stratification in concentric coextrusion of two viscoelastic fluids. J. Non-Newtonian Fluid Mech. 40: 155-175

Chen K P 1993 Wave formation in the gravity-driven low-Reynolds number flow of two liquid films down an inclined plane. Phys. Fluids A 5(12): 3038-3048

Chokshi P and Kumaran V 2008 Weakly nonlinear stability analysis of flow past a neo-Hookean solid at arbitrary Reynolds number. Phys. Rev. E 77(056): 303

Demekhin E A, Kalliadasis S and Velarde M G 2006 Suppressing falling film instabilities by Marangoni forces. Phys. Fluids 18: 042,111 (1-16)

Gaurav and Shankar V 2009 Stability of fluid flow through deformable neo-Hookean tubes. J. Fluid Mech. 627: 291-322

Gaurav and Shankar V 2010a Role of wall deformability on interfacial instabilities in gravity-driven twolayer flow with a free surface. Phys. Fluids 22: 094,103

Gaurav and Shankar V 2010b Stability of pressure-driven flow in a deformable neo-Hookean channel. J. Fluid Mech. 659: 318-350

Gaurav and Shankar V 2013 Manipulation of instabilities in core-annular flows using a deformable solid layer. Phys. Fluids 25: 014,104

Gkanis V and Kumar S 2003 Instability of creeping Couette flow past a neo-Hookean solid. Phys. Fluids 15: 2864-2471

Huang C and Khomami B 2001 Role of dynamic modulation on stability of multilayer Newtonian and viscoelastic flows down an inclined plane. J. Non-Newtonian Fluid Mech. 97(1): 67-86 
Jain A and Shankar V 2007 Instability suppression in viscoelastic film flows down an inclined plane lined with a deformable solid layer. Phys. Rev. E 76: 046,314 (1-14)

Jain A and Shankar V 2008 Elastohydrodynamic suppression of free-surface instabilities in annular liquid film flow outside wires and inside tubes. Ind. Engg. Chem. Res. 47: 6473-6485

Kumaran V 2000 Classification of instabilities in flow past flexible surfaces. Current Sci. 79: 766-773

Kumaran V, Fredrickson G H and Pincus P 1994 Flow induced instability of the interface between a fluid and a gel at low Reynolds number. J. Phys. II France 4: 893-904

Lin S P and Chen J N 1997 Elimination of three-dimensional waves in a film flow. Phys. Fluids 9: 39263928

Macosko C 1994 Rheology: Principles, measurements, and applications. VCH, New York

Preziosi L, Chen K P and Joseph D D 1989 Lubricated pipelining: stability of core-annular flow. J. Fluid Mech. 201: 323-356

Rayleigh L 1878 On the instability of jets. Proc. London MathSocs 10: 4

Rayleigh L 1892 On the stability of cylinder of viscous liquid under capillary force. Phil. Mag. 34: 145

Renardy Y 1988 Stability of the interface in two-layer Couette flow of upper convected Maxwell liquids. $J$. Non-Newtonian Fluid Mech. 28: 99-115

Shankar V 2005 Stability of two-layer viscoelastic plane Couette flow past a deformable solid layer: Implications of fluid viscosity stratification. J. Non-Newtonian Fluid Mech. 125: 143-158

Verma M K S and Kumaran V 2013 A multifold reduction in transition Reynolds number, and ultra fast mixing, in a micro-channel due to dynamical instability induced by soft wall. J. Fluid Mech. 727: 407-455

Yih C S 1963 Stability of liquid flow down an inclined plane. Phys. Fluids 6: 321-334

Yih C S 1967 Instability due to viscosity stratification. J. Fluid Mech. 27: 337-350 